\title{
Estrategia Psicopedagógica para la reducción de la disortografía en estudiantes de secundaria
}

\author{
Psychopedagogical Strategy for the reduction of dysorthography in secondary \\ school students
}

\author{
Estratégia psicopedagógica para a redução da disortografia em alunos \\ do ensino médio
}

\author{
Zuriel Jeremías Gonzáles Burgoa \\ zurieljere@hotmail.com \\ https://orcid.org/0000-0003-4882-0895 \\ Universidad Privada Domingo Savio, Potosí, Bolivia
}

Recibido: noviembre 2019 Revisado: noviembre 2019 Aceptado: diciembre 2019 Publicado: enero 2020

\section{RESUMEN}

La disortografía es la dificultad para producir textos escritos correctos y coherentes, las consecuencias de esta condición son el bajo rendimiento y la desmotivación del estudiante ocasionando altos niveles de deserción y fracaso escolar El artículo presenta la estrategia psicopedagógica para atender la disortografia de los estudiantes de 3 . de secundaria del colegio Otto Felipe Braun" en Potosí, Bolivia. La muestra intencional fue de 45 estudiantes que cursan en el 3. " $A$ " de secundaria y 12 profesores que se desempeñan en este curso. Las técnicas para la recolección de los datos fueron la revisión documental y la encuesta; respectivamente los instrumentos estuvieron constituidos por la matriz de registro y análisis y el cuestionario estructurado. La variable dependiente se operacionalizó en tres dimensiones acentuación, sintaxis y gramática con sus respectivos indicadores. Como resultado se diseñó la estrategia Psicopedagógica con fundamento en los principios de la pedagogía, la psicología y la didáctica.

Palabras clave: Disortografía; estrategia psicopedagógica; secundaria; escritura

\begin{abstract}
Dysorthography is the difficulty to produce correct and coherent written texts, the consequences of this condition are low performance and the lack of motivation of the student, causing high levels of dropout and school failure. The article presents the psych pedagogical strategy to deal with the dysorthography of 3rd year high school students at the "Otto Felipe Braun" School in Potosí, Bolivia. The intentional sample consisted of 45 students who attend the 3rd " $A$ " of secondary school and 12 teachers who work in this course. The techniques for data collection were the documentary review and the survey; respectively, the instruments consisted of the registration and analysis matrix and the structured questionnaire. The dependent variable was operationalized in three dimensions: accentuation, syntax, and grammar with their respective indicators. As a result, the Psych pedagogical strategy was designed based on the principles of pedagogy, psychology and didactics.
\end{abstract}

Key words:

Dysorthography; psych pedagogical strategy; secondary school; writing
RESUMO

A disortografia é a dificuldade de se produzir textos escritos corretos e coerentes, as consequências dessa condição são o baixo desempenho e a falta de motivação do aluno, ocasionando altos índices de evasão e fracasso escolar. $\mathrm{O}$ artigo apresenta a estratégia psicopedagógica para atender a disortografia dos alunos do $3^{\circ}$ ano do ensino médio da Escola Otto Felipe Braun "em Potosí, Bolívia. A amostra intencional foi composta por 45 alunos que frequentam o $3^{\circ}$ " $A$ " do ensino médio e 12 professores que atuam neste curso. As técnicas de coleta de dados foram a revisão documental e o survey; respectivamente, os instrumentos constituíram-se da matriz de registro e análise e do questionário estruturado. A variável dependente foi operacionalizada em três dimensões: acentuação, sintaxe e gramática com seus respectivos indicadores. Como resultado, a estratégia psicopedagógica foi desenhada com base nos princípios da pedagogia, da psicologia e da didática.

Palavras-chave:

Disortografia; estratégia psicopedagógica; secundário; escrita 


\section{INTRODUCCIÓN}

La escritura es una destreza psicomotriz mediante la cual se aprende a escribir palabras y oraciones, a medida que se ejercite de forma sistemática y progresiva mayor será la velocidad y legibilidad en la producción de los textos. Aprender a escribir es más que transcribir sonidos a grafemas sino que implica la capacidad para escribir no solo palabras sino textos coherentes ya que la verdadera función de la escritura es comunicar un mensaje escrito. (Fernández, Díaz, Bueno, Cabañas y Jiménez (2017).

La disortografía se define como un problema específico de la escritura en la cual se muestran dificultades para escribir de forma correcta las palabras. Autores como García (1989) y Celdrán y Zamorano (2017) concuerdan que en los sujetos con disortografía tienen problemas para realizar la correcta asociación entre el código escrito, las reglas ortográficas y la escritura de las palabras.

Tener el conocimiento de las causas que pueden ocasionar esta dificultad permite comprender mejor el problema. Esteve y Jiménez, (1988) las resumen por su origen como: Perceptivas, intelectuales, lingüísticas, afectivo-emocionales y las pedagógicas; cabe destacar que estas últimas aparecen cuando el método de enseñanza de la escritura no es adecuado o no se ajusta a las necesidades específicas del alumno.

Una de las consecuencias de la disortografía es el bajo rendimiento y la desmotivación del estudiante ocasionando altos niveles de deserción y fracaso escolar. Mateos y López (2011) reportan como resultado de sus investigaciones que si no se le presta la debida atención a esta dificultad vamos a encontrar a niños y adolescentes que presentarán después: fracaso escolar, problemas emocionales, trastornos comportamentales, problemas de adaptación familiar, laboral y/o social.
Por lo anteriormente expuesto, es importante atender las dificultades en la escritura que presentan los estudiantes de $3 .^{\circ}$ de secundaria, para lo cual se plantea el problema y objetivo de la investigación.

\section{Problema}

¿Cuáles con los elementos que deben constituir una estrategia Psicopedagógica que permita la reducción de la disortografía en estudiantes de $3 .^{\circ}$ " $A$ " de secundaria del colegio "Otto Felipe Braun" en Potosí, Bolivia?

\section{Objetivo}

Diseñar una estrategia Psicopedagógica que permita la reducción de la disortografía en estudiantes de 3. " $A$ " de secundaria del colegio “Otto Felipe Braun” en Potosí, Bolivia.

\section{MÉTODO}

En atención al objetivo declarado fue una investigación proyectiva cuyo producto es el diseño de la propuesta de una estrategia didáctica fundamentada en el diagnóstico de necesidades. El enfoque es mixto porque se obtienen y procesan datos de forma cualitativa y cuantitativa, el diseño es de campo, pues los datos se recolectaron en el contexto natural.

El estudio se enmarcó en el colegio "Otto Felipe Braun", ubicado al norte del departamento de Potosí, en la provincia Tomas Frías en Bolivia. Esta institución atiende 815 estudiantes, en los niveles desde $3 .^{\circ}$ hasta $6 .^{\circ}$ de secundaria, con un equipo de 42 docentes en sus diferentes especialidades.

La muestra intencional la conforman 45 estudiantes que cursan en el 3. " $A$ " de secundaria y 12 profesores que se desempeñan en este curso. La edad de los estudiantes oscila entre 13 y 14 años. 
Algunos de los alumnos manifiestan que deben trabajar para contribuir con el sustento económico de sus familias.

Las técnicas para la recolección de los datos fueron la revisión documental y la encuesta; respectivamente los instrumentos estuvieron constituidos por la matriz de registro y análisis y el cuestionario estructurado.

La matriz quedó conformada por 16 ítems, esta matriz se utilizó para registrar y analizar los 45 cuadernos de apuntes en borrador, pertenecientes a los estudiantes, lo cual permitió diagnosticar el nivel de disortografia de la muestra, discriminando las tres dimensiones de la variable acentuación, sintaxis y gramática con sus respectivos indicadores (Ver Cuadro 1).

El cuestionario estructurado con 16 ítems se aplicó a 12 docentes para diagnosticar la disortografía de los estudiantes desde la percepción de sus profesores.

La variable dependiente de la investigación es la disortografía y su operacionalización con tres dimensiones se observa en el Cuadro 1.

Cuadro 1. Operacionalización de la variable dependiente.

\begin{tabular}{|c|c|c|}
\hline Definición operativa & Dimensión & Indicadores \\
\hline \multirow{16}{*}{$\begin{array}{l}\text { Dificultad en la escritura } \\
\text { cuya característica principal } \\
\text { es un déficit específico y } \\
\text { significativo de la ortografía }\end{array}$} & \multirow[t]{3}{*}{ Acentuación } & Omisión de tilde en palabras agudas \\
\hline & & Omisión de tilde en palabras esdrújulas \\
\hline & & Omisión de tilde en palabras sobresdrújulas \\
\hline & \multirow[t]{7}{*}{ Sintaxis } & Omisión de género \\
\hline & & Omisión de número \\
\hline & & Omisión de tiempo \\
\hline & & Omisión de persona \\
\hline & & Incoherencia del texto \\
\hline & & Omisión de conjunciones \\
\hline & & Omisión de preposiciones \\
\hline & \multirow[t]{6}{*}{ Gramática } & Omisión de signos de puntuación \\
\hline & & Omisión de grafemas \\
\hline & & Adición de grafemas \\
\hline & & Omisión de palabras \\
\hline & & Adición de palabras \\
\hline & & Omisión de la letra “h” \\
\hline
\end{tabular}

\section{RESULTADOS}

Las derivaciones se presentan de acuerdo al instrumento utilizado para su recolección. La matriz de registro permitió diagnosticar la disortografía de los estudiantes, se aplicó a los 45 cuadernos de apuntes en borrador de cada estudiante. El cuestionario se le aplicó a los docentes para conocer desde su percepción la disortografía de los estudiantes. 


\section{Matriz de registro}

La primera dimensión mide la acentuación, en el gráfico 1 se plasman los resultados.

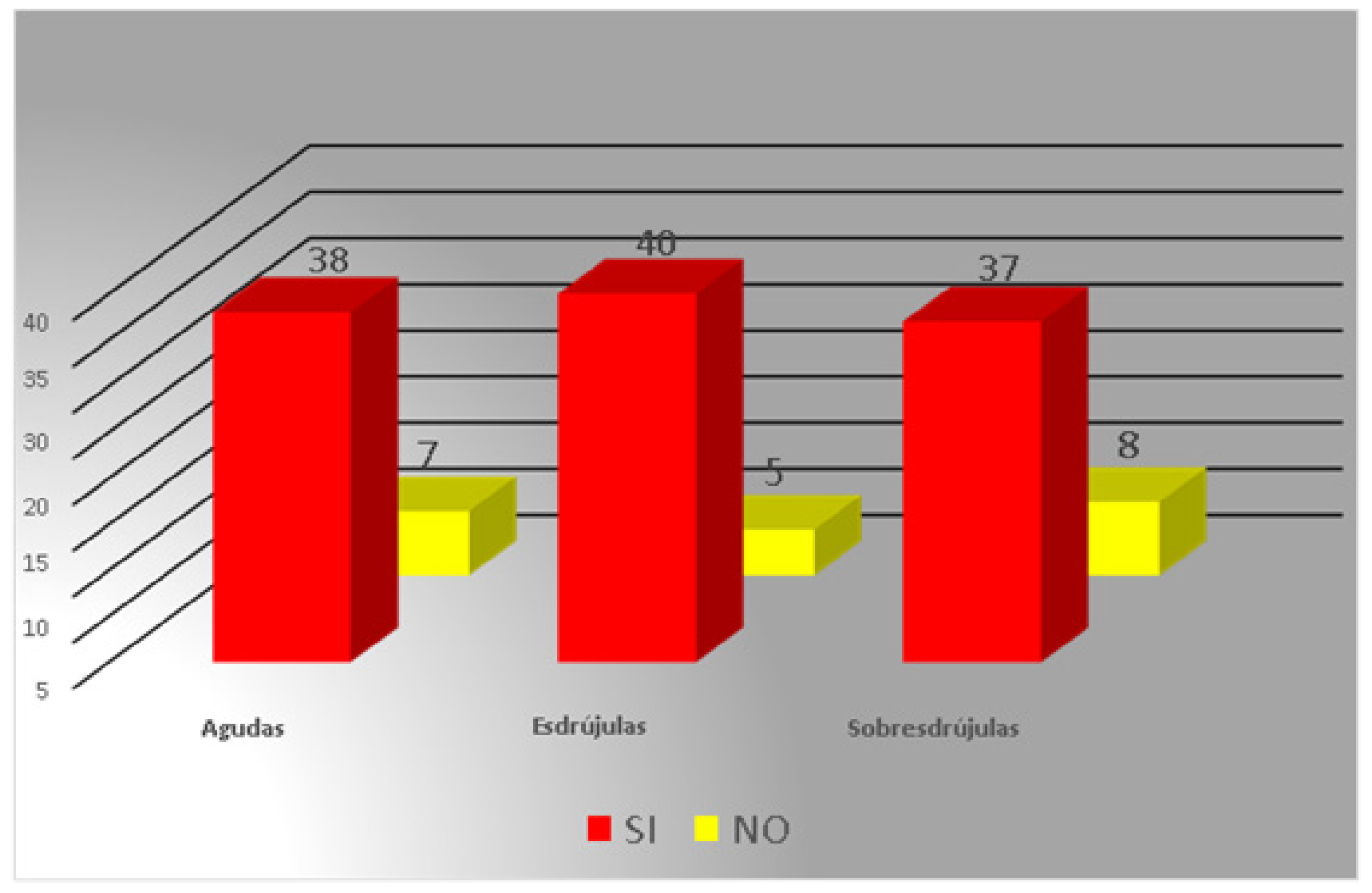

Gráfico 1. Omisión de la tilde.

El $84 \%$ que equivalen a 38 estudiantes omiten la tilde en las palabras agudas, solo el $16 \%$ utiliza la tilde en estas palabras. El $89 \%$ que representa a 40 estudiantes omiten la tilde en las palabras esdrújulas y en las sobresdrújulas la situación es similar el $82 \%$ que son 37 estudiantes omiten la tilde en estas palabras el $18 \%$ no la omite.

La acentuación es imprescindible en la habilidad para escribir correctamente y de manera comprensible para que los estudiantes puedan expresarse correctamente en sus textos escritos.
Estos índices de omisión son muy altos, en este nivel los estudiantes deberían tener mejor uso de la tilde

La segunda dimensión permite el diagnóstico de la sintaxis, que es la relación y orden de las palabras. En los cuadernos de apuntes de los estudiantes se encontró problemas en esta dimensión, con respecto al género, número, persona, tiempo verbal, comprensión del texto, uso de conjunciones y de preposiciones. Los resultados se muestran en el Gráfico 2. 


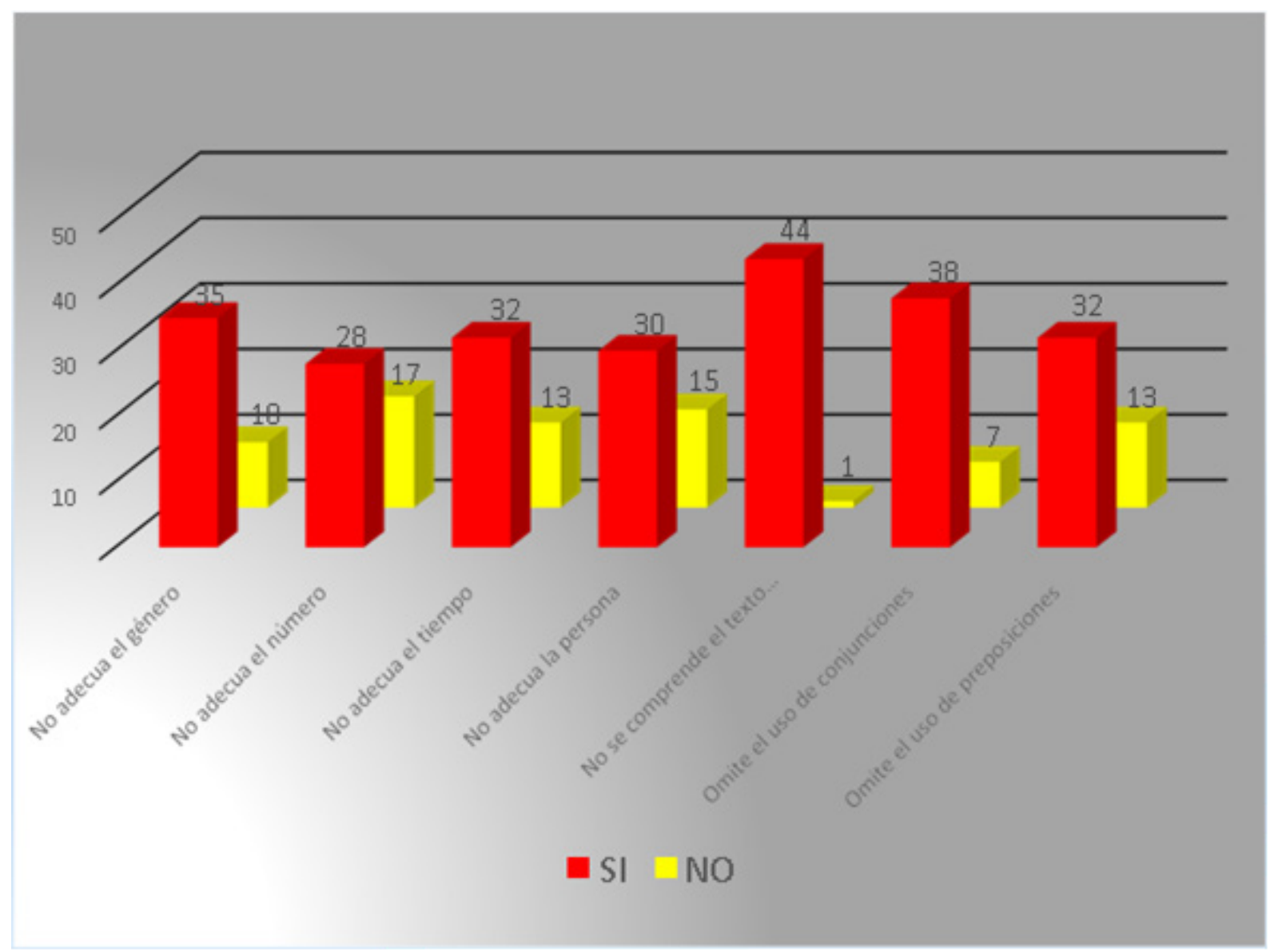

Gráfico 2. Omisión de los elementos de la sintaxis.

Los estudiantes presentan grandes fallas en la sintaxis de sus textos, los resultados, que oscilan entre el $98 \%$ y el $67 \%$ muestran que los estudiantes tienen una sintaxis muy baja.

En una composición escrita un estudiante debe aplicar tanto artículo como sustantivo con el mismo género sea este masculino o femenino, sin embargo 35 estudiantes confunden el uso del género entre un sustantivo y su artículo.

En el segundo indicador 28 estudiantes no usan de manera correcta el plural y singular en los textos que realiza, presentan confusión entre los artículos y sus respectivos sustantivos.

El tercer indicador muestra que 32 estudiantes usan incorrectamente el tiempo pasado, presente y futuro; en un mismo párrafo utilizan varios tiempos verbales.
En el cuarto indicador 30 estudiantes muestran dificultades para mantener la redacción de forma coherente, pasan indistintamente de una narración en primera a tercera persona o viceversa.

El sexto indicador evidencia que 44 estudiantes presentan varias incoherencias de todo tipo, por lo tanto sus textos no son comprensibles.

En los dos últimos indicadores 38 estudiantes no usan correctamente las conjunciones y 32 no usan adecuadamente las preposiciones.

La tercera dimensión de la disortografía es la gramática, se identifica la omisión de signos de puntuación, grafemas, palabras y la letra h; así como también la adición de grafemas y palabras (Ver Gráfico 3). 


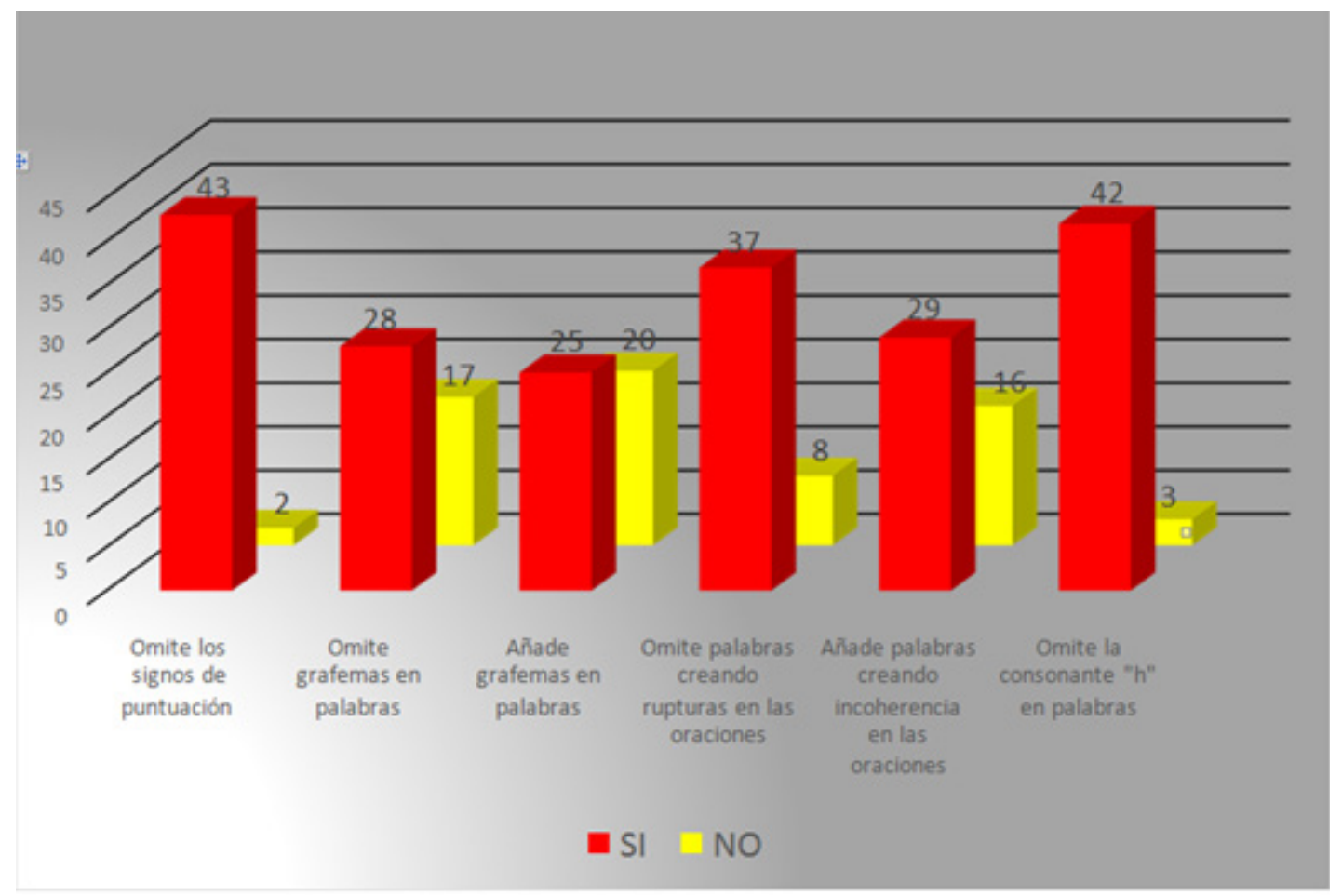

Gráfico 3. Omisión de elementos ortográficos.

Los indicadores en los cuales los estudiantes presentan mayores debilidades, entre el $96 \%$ y el $82 \%$, omiten: signos de puntuación, palabras y la letra h. El $64 \%$ añade palabras y más del 50\% omiten o añaden grafemas.

Estos resultados indican las deficiencias en cuanto al área gramatical, las consecuencias se observan en los escritos de los estudiantes los cuales son difíciles de comprender por la falta de coherencia.

\section{Cuestionario a los docentes}

El cuestionario que respondieron los 12 docentes que atienden a los estudiantes de tercero de secundaria, coinciden con los resultados de la matriz de registro con la cual se analizaron los cuadernos de apuntes.

Los docentes manifiestan que los estudiantes presentan dificultades en la acentuación, sintaxis y gramática y agregan que esto trae como consecuencia una baja calidad en sus escritos, un bajo rendimiento y desmotivación por el estudio.
Todos están de acuerdo que es necesario diseñar y aplicar una estrategia pedagógica que permita atender esta situación para contribuir a mejorar los aspectos de la escritura.

\section{Propuesta}

El diseño de la propuesta para atender la disortografía se fundamenta en la contribución de varias ciencias como la Psicología, la Pedagogía y la Didáctica. Los principios de la Psicología que se consideran son los de la motivación, la creatividad y la comunicación. El aporte de la pedagogía es la comprensión de la educación como un proceso integral en el cual se conjugan lo social y lo individual, lo afectivo y lo cognitivo, lo consciente y lo involuntario. Desde la didáctica el principio de autonomía y atender la integralidad desde el saber, el hacer y el ser del estudiante. (Córdoba, 2017; Gia, 2017, Restrepo, 2008; Universidad de Ciencias Pedagógicas “Manuel Ascunce Domenech”, 2010 y Piaget, 1991). 


\section{Estrategia psicopedagógica para la atención de la disortografía}

\section{Objetivo}

- Mejorar la escritura de los estudiantes de tercero de secundaria que presentan disortografía.

- Desarrollar los prerrequisitos implicados en el proceso de la escritura.

- Desarrollar habilidades grafo motrices.

- Lograr el uso correcto de los signos de puntuación.

\section{Metodología}

La estrategia didáctica está constituida por 9 talleres y 6 sesiones de trabajo, que se deben ejecutar en grupos pequeños de trabajo, entre 4 y 6 integrantes, para asegurar la interacción y atención para cada estudiante. El facilitador debe promover la participación de forma activa en el proceso de aprendizaje.

Dirigido a los 45 estudiantes del tercero de secundaria del Colegio "Otto Felipe Braun" situado en la ciudad de Potosí en Bolivia.

A continuación se presenta un resumen de la propuesta:

Taller 1. La ortografía y su importancia.

Tema del taller: Sensibilización acerca de la importancia de la ortografía

Participantes: 45 estudiantes

Fecha y lugar: Colegio Otto Felipe Braun

\begin{tabular}{lll}
\hline Horario & Actividades & Materiales \\
necesarios
\end{tabular}




\section{El animómetro.}

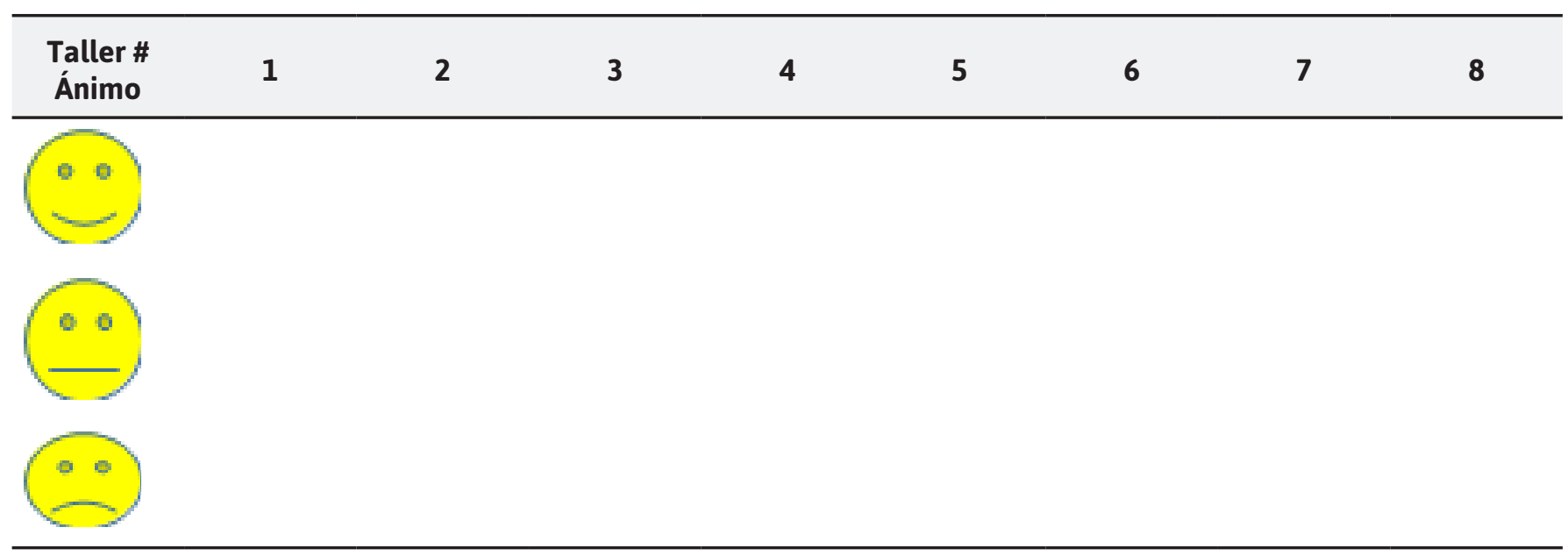

Taller 2. Acentuación de palabras.

Tema del taller: Las palabras agudas y graves o llanas

Participantes: 45 estudiantes

Fecha y lugar: Colegio Otto Felipe Braun

\begin{tabular}{|c|c|c|}
\hline Horario & Actividades & $\begin{array}{l}\text { Materiales } \\
\text { necesarios }\end{array}$ \\
\hline $15 \mathrm{~min}$. & $\begin{array}{l}\text { Fase inicial } \\
\text { - Bienvenida. Dinámica llamada, dibujando un autorretrato, se pide a los participantes que } \\
\text { dibujen un autorretrato y que incluyan tres razones por las que participan del taller. } \\
\text { - } \quad \text { Expectativas del taller tanto del facilitador como de los estudiantes. } \\
\text { - Objetivo: Concientizar a los estudiantes acerca del uso de las tildes en las palabras agudas } \\
\text { y graves o llanas. }\end{array}$ & \\
\hline $5 \mathrm{~min}$. & $\begin{array}{l}\text { Fase central } \\
\text { Presentación de los contenidos: } \\
\text { - } \quad \text { mportancia de la buena ortografía. } \\
\text { - Importancia de la comunicación escrita eficaz y correcta. }\end{array}$ & $\begin{array}{l}\text { - Pizarra } \\
\text { - Tizas } \\
\text { - Almohadilla }\end{array}$ \\
\hline $5 \mathrm{~min}$. & Receso & \\
\hline $45 \mathrm{~min}$. & $\begin{array}{l}\text { Dinámica: Phillips 6-6. La que consiste en dividir a todo el grupo en grupos de } 6 \text { presentando } \\
\text { el tema del taller en forma de interrogante a cada grupo, se les da un papelógrafo para } \\
\text { que después de } 6 \text { minutos cada grupo exponga la importancia de la ortografía en su medio. } \\
\text { Trabajar la temática con los participantes en grupos. Realizar la plenaria. Después de cada } \\
\text { uno de los aportes de los grupos se explica los dos temas preparados teniendo en cuenta } \\
\text { los aportes de los estudiantes. }\end{array}$ & $\begin{array}{l}\text { - Papelógrafo } \\
\text { - Marcadores }\end{array}$ \\
\hline $5 \mathrm{~min}$. & Receso & \\
\hline $45 \mathrm{~min}$. & $\begin{array}{l}\text { Fase final Evaluación de la dinámica } \\
\text { - Se realiza una retroalimentación del tema por medio de preguntas al azar hechas por el } \\
\text { facilitador. } \\
\text { - El facilitador presenta las conclusiones de la temática presentada. } \\
\text { - Evaluación, como evaluación final se marca el animómetro al salir del aula. Otro } \\
\text { instrumento para evaluar constantemente cada taller es el diario de registro anecdótico. }\end{array}$ & - Animómetro \\
\hline
\end{tabular}


Tema del taller: Las palabras agudas y graves o llanas

Participantes: 45 estudiantes

Fecha y lugar: Colegio Otto Felipe Braun

Horario

Actividades

$15 \mathrm{~min} . \quad$ Fase final

- Se realiza una retroalimentación del tema por medio de preguntas al azar hechas por el facilitador.

- El facilitador presenta las conclusiones de la temática presentada.

- Evaluación, como evaluación final del taller se realiza una lluvia de ideas acerca de los dos conceptos estudiados y cómo se aplicarían a la vida diaria de los estudiantes en su proceso de enseñanza aprendizaje, después se marca el animómetro al salir del aula como evaluación global de todos los días.

Taller 3. Acentuación de palabras.

Tema del taller: Las palabras esdrújulas y sobresdrújulas

Participantes: 45 estudiantes

Fecha y lugar: Colegio Otto Felipe Braun

\begin{tabular}{|c|c|c|}
\hline Horario & Actividades & $\begin{array}{l}\text { Materiales } \\
\text { necesarios }\end{array}$ \\
\hline $15 \mathrm{~min}$. & $\begin{array}{l}\text { Fase inicial } \\
\text { - Bienvenida. Dinámica denominada escribe en el aire, se realiza por medio de un sorteo por } \\
\text { número al azar, entonces al que le toque sale al frente del grupo y escribe su nombre de } \\
\text { forma imaginaria en el aire, pensando en escribir en un pizarrón. } \\
\text { - } \quad \text { Expectativas del taller tanto del facilitador como de los estudiantes. } \\
\text { Objetivo: Concientizar a los estudiantes acerca del uso de las tildes en las palabras } \\
\text { esdrújulas y sobresdrújulas. }\end{array}$ & \\
\hline $5 \mathrm{~min}$. & $\begin{array}{l}\text { Fase central } \\
\text { Presentación de los contenidos: } \\
\text { - } \quad \text { Las palabras esdrújulas } \\
\text { - Las palabras sobresdrújulas }\end{array}$ & $\begin{array}{l}\text { - Pizarra } \\
\text { - Tizas } \\
\text { - Almohadilla }\end{array}$ \\
\hline $5 \mathrm{~min}$. & Receso & \\
\hline $45 \mathrm{~min}$. & $\begin{array}{l}\text { Dinámica: Para iniciar se forman } 5 \text { grupos al azar de } 9 \text { estudiantes, la dinámica se denomina } \\
\text { lectura comentada, consiste en la entrega de un texto referido al tema a los grupos de } \\
\text { trabajo con el objetivo de que efectúen una lectura individual y una discusión en grupo } \\
\text { para presentar a la plenaria tanto el contenido como el resultado de la discusión en grupo. }\end{array}$ & $\begin{array}{l}\text { - Papelógrafo } \\
\text { - Marcadores }\end{array}$ \\
\hline $5 \mathrm{~min}$. & Receso & \\
\hline $15 \mathrm{~min}$. & $\begin{array}{l}\text { Fase final } \\
\text { - Se realiza una retroalimentación del tema por medio de preguntas al azar hechas por el } \\
\text { facilitador, para ello se lanza una pelota a cualquier grupo y deben dar a conocer lo que } \\
\text { les pareció más interesante del tema. } \\
\text { - El facilitador presenta las conclusiones de la temática presentada. } \\
\text { - Evaluación, como evaluación final del taller se realiza una lluvia de ideas acerca de los } \\
\text { dos conceptos estudiados y cómo se aplicarían a la vida diaria de los estudiantes en su } \\
\text { proceso de enseñanza aprendizaje, después se marca el animómetro al salir del aula como } \\
\text { evaluación global de los talleres. }\end{array}$ & $\begin{array}{l}\text { - Animómetro } \\
\text { - Pelota }\end{array}$ \\
\hline
\end{tabular}


Taller 4. Género y número.

Tema del taller: El género y número en la oración

Participantes: 45 estudiantes

Fecha y lugar: Colegio Otto Felipe Braun

\begin{tabular}{llr}
\hline Horario & Actividades & Materiales \\
& necesarios
\end{tabular}

$15 \mathrm{~min} . \quad$ Fase inicial

- Bienvenida. Dinámica denominada nombres y adjetivos, los participantes deben pensar en un adjetivo para describir cómo se sienten y cómo están. El adjetivo debe empezar con la misma letra que sus nombres. Por ejemplo, mi nombre es Ernesto y estoy enojado, soy tito y soy tímido; así, hasta terminar.

- Expectativas del taller tanto del facilitador como de los estudiantes.

- Objetivo: Fortalecer los conocimientos acerca de la segunda dimensión sintaxis específicamente género y número.

5 min. $\quad$ Fase central

Presentación de los contenidos:

- El género masculino y femenino en la oración.

- Pizarra

- El número singular y plural en la oración.

5 min. Receso

45 min. - Dinámica: Cambiando el género y número a la oración. Para empezar, se da la consigna que a cada pregunta que se dé en singular la respuesta sea en plural, y además que si la pregunta o respuesta tiene género masculino se lo transforme en femenino y viceversa. Por ejemplo: si la pregunta es ¿cuál es tu nombre? y su nombre es Carlos la respuesta será, mis nombres son Carlas y se habrá cambiado tanto género como número a la respuesta.

- Para trabajar la temática con los estudiantes se utiliza el papelógrafo y marcadores y se desarrolla el contenido de forma expositiva.

\section{5 min. $\quad$ Receso}

15 min. Fase final

- Se realiza una retroalimentación. Con preguntas puntuales acerca de cuál es la importancia al momento de escribir y en qué forma lo aplican a su proceso de aprendizaje.

- El facilitador presenta las conclusiones de la temática.

- Evaluación, para esto se usa la técnica de lluvia de ideas, esperando a que, de forma voluntaria un estudiante se ponga en pie y pueda dar su opinión acerca de lo que aprendió, lo cual se anota en un registro. Después se marca el animómetro al salir del aula como evaluación global de los talleres. 
Taller 5. Tiempo verbal y persona.

Tema del taller: El tiempo y la persona

Participantes: 45 estudiantes

Fecha y lugar: Colegio Otto Felipe Braun

\begin{tabular}{lll}
\hline Horario & Actividades & Materiales \\
& & necesarios \\
\hline
\end{tabular}

$15 \mathrm{~min}$ Fase inicial

- Bienvenida. Dinámica denominada ¿Quién es el líder? Los estudiantes se sientan formando un círculo. Uno de ellos se ofrece como voluntario y sale del curso entre tanto el resto escoge un líder, el líder debe hacer una serie de acciones como aplaudir, zapatear y más, que los demás imitan, después el voluntario ingresa y se para en el centro y busca al líder que cambia las acciones; termina cuando encuentra al líder y él sale y los otros escogen otro líder.

- Expectativas del taller del facilitador.

- Objetivo: Fortalecer los conocimientos acerca de la segunda dimensión sintaxis específicamente tiempo y persona.

5 min. $\quad$ Fase central

Presentación de los contenidos:

- Los tiempos verbales en la oración.

Pizarra

- Tizas

- La 1ra, 2da y 3ra persona en la oración.

\section{5 min. $\quad$ Receso}

45 min. - Dinámica: Después de dar a conocer la temática los estudiantes realizan sociodramas acerca de la familia con variantes distintas, unos solo deberán hablar en tiempo pasado Ira persona, otros en presente y 2 da persona y el último grupo en futuro y 3ra persona aplicando lo que aprendieron en la exposición de la temática.

- Trabajar la temática con los participantes en tres grupos distintos escogidos por afinidad de los estudiantes, se les indica que los grupos se reunirán después de la exposición de la temática y se les dará consignas distintas.

\section{5 min. $\quad$ Receso}

15 min. Fase final

- Se realiza una retroalimentación, preguntando a cada grupo que dé su opinión acerca de lo que hicieron los otros dos grupos.

- El facilitador presenta las conclusiones de la temática presentada.

- Evaluación, para empezar, se evalúa lo aprendido por medio de preguntas que realiza el facilitador acerca de la temática y después se marca el animómetro al salir del aula como evaluación diaria de los talleres. 
Taller 6. Signos de puntuación.

Tema del taller: Los signos de puntuación.

Participantes: 45 estudiantes

Fecha y lugar: Colegio Otto Felipe Braun

\begin{tabular}{llr}
\hline Horario & Actividades & Materiales \\
& & necesarios \\
\hline
\end{tabular}

\section{5 min. Fase inicial}

- Bienvenida. Dinámica denominada Me Voy de Viaje, se organizan en círculo y el facilitador puede empezar diciendo me voy de viaje y me llevo un abrazo y da un abrazo al estudiante de su derecha, después él debe repetir las palabras y la acción y agregar una nueva acción y así hasta terminar todos.

- Expectativas del taller dadas por el facilitador.

- Objetivo: Fortalecer los conocimientos de los estudiantes acerca de la importancia de los signos de puntuación.

$5 \min$.

Fase central

Presentación de los contenidos:

- La utilización e importancia de los signos de puntuación.
- Pizarra

- Tizas

- Almohadilla

\section{5 min. $\quad$ Receso}

45 min. - Dinámica: Palabras Cruzadas, se realiza en una hoja bond elaborada anteriormente, los estudiantes deben agruparse en grupos de 9 para no demorar con la solución, deben rellenar una parrilla con palabras que se tendrán que deducir de las instrucciones dadas mediante una definición, la utilización de sinónimos o de antónimos, contestando preguntas o adivinanzas. Cada una de las casillas corresponde a una letra de la palabra. Habrá palabras en posición vertical y horizontal.

- Trabajar la temática con los participantes en grupos de 9 por medio de una lectura comentada del tema que se presenta.

\section{5 min. Receso}

15 min. Fase final

- Se realiza una retroalimentación dada por el facilitador de la importancia del uso de los signos de puntuación.

- $\quad$ El facilitador presenta las conclusiones de la temática.

- Evaluación, para la fase final, el facilitador lanza una pelota al azar a cualquier participante para que responda a preguntas puntuales acerca de la importancia de los signos de puntuación al momento de escribir. Después se marca el animómetro. 
Taller 7. La muda sí importa.

Tema del taller: La muda sí importa

Participantes: 45 estudiantes

Fecha y lugar: Colegio Otto Felipe Braun

\begin{tabular}{llr}
\hline Horario & Actividades & Materiales \\
& & necesarios \\
\hline
\end{tabular}

\section{5 min. Fase inicial}

- Bienvenida. Dinámica denominada mensajes confusos, los estudiantes se sientan en círculo, y el facilitador pasa un mensaje largo en voz baja a la oreja del primer estudiante del círculo y después éste al de su derecha hasta terminar, cuando llegue el mensaje al final se debe comparar con el mensaje dado al principio, el resultado será un gracioso mensaje confuso.

- Expectativas del taller dadas por el facilitador.

- Objetivo: fortalecer los conocimientos de los estudiantes acerca del uso de la $\mathrm{H}$ en las oraciones.

5 min. $\quad$ Fase central

Presentación de los contenidos:

- Pizarra

- Uso e importancia de la $\mathrm{H}$

- Tizas

- Almohadilla

\section{5 min. $\quad$ Receso}

$45 \mathrm{min.} \quad$ - Dinámica: El dado del conocimiento, se pide 6 voluntarios a los que se les muestra 6 preguntas y se les da un dado que deben lanzar y según el número que le salga debe responder a la pregunta que tenga el mismo número.

- Trabajar la temática con los participantes, para esto se pide que los estudiantes digan números del uno al cinco varias veces, terminando se pide que todos lo que dijeron uno se unan, los dos y los tres y los cuatro y finalmente los cinco, después de conformar los grupos deberán socializar la temática que se tiene en fotocopias y compartir apreciaciones al respecto con los demás grupos.

\section{5 min. $\quad$ Receso}

15 min. Fase final

- El facilitador presenta las conclusiones de la temática presentada.

Animómetro

- Papelógrafo

- Evaluación, para esta fase se pide que cada grupo pase a la mesa del otro grupo de la derecha, cuando estén así, cada uno debe explicar lo que el otro grupo dijo anteriormente intentando reproducir aquello que los otros dijeron en un papelógrafo acerca del tema. Después se marca el animómetro al salir del aula como evaluación global de los talleres.

\section{- Dados}

- Fotocopias del tema 
Sesión 1. Uso de la acentuación.

Objetivo: Mejorar el uso de la acentuación

Contenido: Palabras agudas y graves o llanas

Recursos: Cartulina, tizas de colores, marcadores
Participantes: 5

Tiempo: $30^{\prime}$

\section{Actividades}

Motivación. Para iniciar la sesión a modo de introducción se realiza un ejercicio llamado había una pulga, consiste en cantar una canción y el estudiante deberá repetirla, para complicarlo se repite la misma canción, pero usando solo la vocal "a" en cada una de las palabras de la canción y así ir usando las cinco vocales.

Recuperación de la información. Terminada la dinámica se pregunta al estudiante cuánto recuerda acerca de las palabras agudas y graves o llanas, después de que el estudiante da su respuesta se le da una breve reseña acerca de las reglas para acentuar las palabras.

Conflicto cognitivo. En unas fichas se tiene palabras agudas y graves o llanas, pero escritas de forma incorrecta, lo que debe hacer el estudiante es verlas y si están bien dejarlas y si están mal corregirlas y escribirlas de forma correcta

Procesamiento. Después que corrige las palabras deberá clasificar las mismas en columnas, la primera columna debe ser de las palabras agudas, la segunda columna debe ser de palabras graves o llanas.

Aplicación. Después de clasificar cada una de las palabras y haber reconocido el tipo de palabra según la acentuación deberá realizar oraciones con las mismas palabras.

Reflexión. Después de terminada la actividad de la sesión numero 1 el grupo de estudiantes, realizará la meta cognición de la actividad para determinar que aprendizaje obtuvo y como lo logró.

Evaluación. Se evaluará el proceso del estudiante por medio de sus respuestas durante toda la sesión. Además, se identificará en qué forma realizó la actividad y si la selección de palabras fue correcta.

Sesión 2. Uso de la acentuación.

Objetivo: Mejorar el uso de la acentuación

Participantes: 5

Contenido: Palabras agudas y graves o llanas

Tiempo: $30^{\prime}$

Recursos: Cartulina, tizas de colores, marcadores

\section{Actividades}

Motivación. Para iniciar la sesión a modo de introducción se realiza una dinámica llamada, ¿Quién soy? Consiste en poner nombres de diferentes personas famosas en la espalda de los cinco estudiantes, entre ellos deben preguntarse acerca de su identidad, las preguntas pueden ser respondidas con sí o no, el juego termina cuando ya todos saben quiénes son.

Recuperación de la información. Terminada la dinámica se pregunta al estudiante cuánto recuerda acerca de las palabras agudas y graves o llanas, después de que el estudiante da su respuesta se le da una breve reseña acerca de las reglas para acentuar las palabras.

Conflicto cognitivo. En unas fichas se tiene palabras esdrújulas y sobresdrújulas, pero escritas de forma incorrecta, lo que debe hacer el estudiante es verlas y si están bien dejarlas y si están mal corregirlas y escribirlas de forma correcta. 
Objetivo: Mejorar el uso de la acentuación

Participantes: 5

Contenido: Palabras agudas y graves o llanas

Tiempo: $30^{\prime}$

Recursos: Cartulina, tizas de colores, marcadores

\section{Actividades}

Procesamiento. Después que corrige las palabras deberá clasificar las mismas en columnas, la primera columna debe ser de las palabras esdrújulas, la segunda columna debe ser de palabras sobresdrújulas.

Aplicación. Después de clasificar cada una de las palabras y haber reconocido el tipo de palabra, deberá realizar oraciones con las mismas.

Aplicación. Después de clasificar cada una de las palabras y haber reconocido el tipo de palabra, deberá realizar oraciones con las mismas.

Reflexión. Después de terminada la actividad de la sesión numero 2 el grupo de estudiantes, realizará la meta cognición de la actividad para determinar que aprendizaje obtuvo y como lo logró.

Evaluación. Se evaluará el proceso del estudiante por medio de sus respuestas durante toda la sesión. Además, se identificará en qué forma realizó la actividad y si la selección de palabras fue correcta.

Sesión 3. Género, número y tiempo.

Objetivo: Mejorar la escritura utilizando el género, número y tiempo

Participantes: 5

Contenido:

Tiempo: $30^{\prime}$

Recursos: Hojas fotocopiadas del cuento, hojas en blanco

\section{Actividades}

Motivación. Para iniciar la actividad a modo de introducción se pide al grupo que cada uno repita un trabalenguas que sepa de memoria, y después que lo repite se le pide que lo vuelva a repetir, pero, que las palabras lleven la misma vocal. Y después que repite el trabalenguas se le explica porque las palabras no pueden escribirse con una letra demás o en otro género ya que provoca incoherencia en el texto escrito.

Recuperación de la información. Se pide al estudiante que dé a conocer las definiciones que sepa acerca del género, número y tiempo.

Conflicto cognitivo. Dentro de un texto elaborado previamente se le pide al estudiante que encuentre dos ejemplos de uso de género, número y tiempo verbal.

Procesamiento. Procesamiento. Después que el estudiante encuentra los ejemplos se le pide que haga lo contrario con cada uno de los ejemplos, es decir, en los ejemplos de género lo cambie de masculino a femenino, en el número de plural a singular y así sucesivamente con cada uno de los ejemplos.

Aplicación. Aplicación. Después de haber cambiado cada uno de los ejemplos por su contrario, el estudiante deberá realizar oraciones con cada uno de sus ejemplos

Reflexión. Después de terminadas las oraciones se pide al estudiante que pueda decir en sus propias palabras las definiciones y en qué momento se puede aplicar los conocimientos que acaba de aprender.

Evaluación. Se evaluará el proceso del estudiante por medio de sus respuestas durante toda la sesión. 
Sesión 4. Logogén.

Objetivo: Aplicar la técnica logogén para el uso de la escritura de palabras, frases, oraciones, Participantes: 5 párrafos y textos, mediante la ejecución de fichas de aplicación.

Contenido: Ortografía

Tiempo: 30'

Recursos: Fotocopias de cuento, bolígrafos y hojas en blanco

\section{Actividades}

Motivación. Explicar la técnica de logogén

\section{Recuperación de la información.}

1. Sistema de logogenes: se incluyen representaciones de las palabras en la memoria léxica de los sujetos que son reconocidas cuando la información acústica o visual procedente de los estímulos adquiere un determinado nivel de umbral.

2. Sistema cognitivo: nos proporciona claves semánticas y sintácticas correspondientes a las palabras que están en el sistema de logogenes.

3. Sistema de respuesta: incluye claves de pronunciación y deletreo.

Cada palabra que reconocen se encuentra representada en el léxico mental por un logogén y esta representación actúa como un mecanismo de recogida de información visual o acústica, que va activando progresivamente diferentes palabras del léxico hasta que tenga datos suficientes para identificar la palabra apropiada.

Conflicto cognitivo. Ee presenta una lectura seleccionada así, mientras un estudiante lee o el profesor los demás sigan con la mirada y escucha a la vez

\section{Procesamiento.}

Aplicación. Para dinamizar la clase el docente invita a los estudiantes a participar del maratón "Búsqueda rápida", Usando el texto de la lectura buscan lo más rápido que puedan las palabras que se escriben con c, z y con s.

El estudiante que logre encontrar lo más rápido posible las palabras, es el ganador. Copiar en el cuaderno las palabras buscadas.

Hacer que los alumnos escriban oraciones con estas palabras.

Reflexión. Después de terminada la actividad de la sesión numero 4 el grupo de estudiantes, realizará la meta cognición de la actividad para determinar que aprendizaje obtuvo y como lo logró.

Evaluación. Se evaluará el proceso del estudiante por medio de sus respuestas durante toda la sesión. Para el proceso de evaluación se realizará una lluvia de ideas partiendo de todo lo aprendido en la sesión.

Sesión 5. Ortografía y el significado de las palabras.

Objetivo: Fortalecer la ortografía y significado de palabras.

Participantes: 5

Contenido: Uso del diccionario

Tiempo: $30^{\prime}$

Recursos: Diccionarios, hojas en blanco, bolígrafos

\section{Actividades}

Motivación. Para iniciar la sesión se realiza una dinámica llamada; tierra, agua fuego. Consiste en que los cinco estudiantes formen una columna y se les indica que el lugar en el que están es tierra, a su derecha es agua y a su izquierda fuego, entonces cuando el facilitador dice tierra deben permanecer en su lugar, si dice agua van a un paso a la derecha si dice fuego van dos pasos a la izquierda, pierde aquel que no esté en el lugar adecuado y gana aquel que quede hasta el final. 
Objetivo: Fortalecer la ortografía y significado de palabras.

Participantes: 5

Contenido: Uso del diccionario

Tiempo: $30^{\prime}$

Recursos: Diccionarios, hojas en blanco, bolígrafos

\section{Actividades}

Recuperación de la información. Terminada la dinámica se pregunta a los estudiantes el significado de las tres palabras (tierra, agua o fuego) puede responder cualquiera de los cinco.

Conflicto cognitivo. Al inicio de la dinámica se entrega a cada estudiante un diccionario y se les explica que la actividad se denomina El Esgrima del Conocimiento. Consiste en que los estudiantes sostienen sus diccionarios cerrados y con las manos hacia el frente, cuando el facilitador dice una palabra comienzan la búsqueda el primero que lo encuentra se pone de pie y comienza a leer su significado.

Gana el estudiante que primero llegue a cinco palabras encontradas y leídas correctamente.

Procesamiento. Después de que uno de los estudiantes gana deben intentar recordar la mayor parte de palabras que encontraron y sus significados.

Aplicación. Después de recolectar la mayor parte de palabras y sus respectivos significados desarrollan oraciones con las mismas teniendo en cuenta su forma correcta de escribir y su significado.

Reflexión. Después de terminada la sesión cada estudiante tendrá nuevas palabras en su léxico, sin embargo, se le pregunta cómo podrían usar esas palabras en su vida diaria tanto fuera como dentro del colegio.

Evaluación. Se evaluará el proceso del estudiante por medio de sus respuestas durante toda la sesión.

Sesión 6. Reducir la omisión de grafemas.

Objetivo: Reducir la omisión de grafemas

Contenido: La importancia de los grafemas

Recursos: Fotocopias de sopa de letras, hojas en blanco
Participantes: 5

Tiempo: $30^{\prime}$

\section{Actividades}

Motivación. Para iniciar la sesión se realiza una dinámica de presentación denominada Mi nombre en Acróstico, cada estudiante escribe su nombre de arriba hacia abajo y con cada una de las letras de su nombre debe buscar una virtud o defecto que lo identifique para ello tienen 5 minutos. Después que se cumple el tiempo deben dar a conocer su acróstico y presentarse.

Recuperación de la información. Terminada la dinámica se pregunta al estudiante si alguna vez realizó crucigramas y si en algún momento se dio cuenta que sería difícil resolverlos si en el recuadro no pondrían alguna de las letras.

Conflicto cognitivo. Entonces después de las respuestas recibidas se les presenta el crucigrama para el que se les da un tiempo de 10 minutos para resolverlo.

Procesamiento. Al encontrar todas las palabras los estudiantes deben clasificarlas en distintos grupos que las identifiquen según características específicas, femeninas a un lado y masculinas al otro, o singulares y plurales.

Aplicación. Escoger una de las palabras y descomponerla para realizar un acróstico como en la dinámica inicial. Pero ahora la consigna es que cada una de las palabras que salgan de las letras tengan relación con el significado de la palabra 
Objetivo: Reducir la omisión de grafemas

Contenido: La importancia de los grafemas

\section{Participantes: 5}

Tiempo: $30^{\prime}$

Recursos: Fotocopias de sopa de letras, hojas en blanco

\section{Actividades}

Reflexión. Después de terminada la actividad de la sesión numero 3 el grupo de estudiantes, realizará la meta cognición de la actividad para determinar que aprendizaje obtuvo y como lo logró.

Evaluación. Se evaluará el proceso del estudiante por medio de sus respuestas durante toda la sesión. Además, se identificará en qué forma realizó la actividad y si la respuesta es correcta.

\section{CONCLUSIONES}

La disortografía es muy alta, se evidencia porque la escritura de los estudiantes de la muestra presenta dificultades en el uso de las tildes, la sintaxis y la gramática. Los textos que producen no tienen coherencia y presentan dificultades para ser comprendidos por los docentes u otro lector.

Para dar solución a la problemática se diseñó una estrategia psicopedagógica fundamentada en principios de la psicología, la pedagogía y la didáctica, constituida por talleres participativos y sesiones individualizadas.

Los factores que pueden incidir en la aparición de la disortografia son: (a) problema de percepción visual y auditiva lo que ocasiona omisión, adición, sustitución o inversión de fonemas; (b) Las carencias afectivas emocionales pueden generar baja motivación al estudio, baja autoestima y desconfianza; (c) Las carencias pedagógicas que aparecen cuando el método de enseñanza de la escritura no es adecuado o no se ajusta a las necesidades específicas del alumno.

\section{REFERENCIAS}

Celdrán, M., y Zamorano, F. (2017). Dificultades en la adquisición de la lecto-escritura y otros aprendizajes. Logopedas en los equipos de orientación educativa y psicopedagógica, 1-37. https://www.orientacionandujar.es/wpcontent/uploads/2016/04/DIFICULTADESEN-LA-ADQUISICI\%C3\%93N-DE-LA-LECTOESCRITURA-Y-OTROS-APRENDIZAJES.pdf

Cervera, J. F. y Ygual-Fernández, A. (2006). Una propuesta de intervención en trastornos disortográficos atendiendo a la semiología de los errores. Revista de neurología, 42, 117-126

Córdova, Rogel, V. E. (2017). Plan de estrategias psicopedagógicas dirigidas a docentes desde el enfoque constructivista aplicadas a estudiantes con bajo rendimiento. Machala, Ecuador: Universidad Técnica de Machala

Esteve, M. y Jiménez, J. (1988). La disortografía en el aula. (EGB) (EUP). Serie Colección Educación Especial

Fernández, M., Díaz, G., Bueno, P., Cabañas, C. y Jiménez, M. (2017). Dificultades en el aprendizaje de la lectoescritura. España: Magisterio de Educación Primaria. https://campus.autismodiario. com/wp-content/uploads/2019/02/ DificultadesMatematicasLenguaje.pdf

García, J. (1989) Manual de dificultades de aprendizaje. Madrid: Narcea

GiaCorrea, B.E.(2017). Estrategiaspsicopedagógicas para fomentar la participación activa en los estudiantes con problemas de cálculo y baja autoestima. Machala, Ecuador: Universidad Técnica de Machala

Martín Macías, E. M. (2010). Orientaciones para trabajar la escritura. Pedagogía Magna, 8, 9499 
Mateos, R., y López, C. (2011). Problemas de diagnóstico tardío y/o del infradiagnóstico. Revista Educación Inclusiva, 103-111

Piaget, J. (1991). Seis estudios de psicología. España: Labor, S.A

Restrepo, M. (2008). Sistematización de una intervención pedagógica con niños escolares que presentan desórdenes en la expresión escrita. Pereira
Universidad de Ciencias Pedagógicas "Manuel Ascunce Domenech". (2010). Una concepción de la pedagogía como ciencia desde el enfoque histórico cultural. Ciego de Ávila: Centro de estudio e investigación "José Martí” 